\title{
Toward a history of explanation in science communication: the case of Madame Wu experiment on parity violation
}

\section{Eugenio Bertozzi}

Abstract

Keywords

Introduction
By focusing on a specific episode of $20^{\text {th }}$ Century physics - the discovery of parity violation in 1957 - the paper presents a study of the types of explanations of the crucial experiment as they are found in different editorial categories: a peer-review journal, a popular science book, an encyclopedia and a newspaper articles. The study provides a fine-grained description of the mechanism of the explanation as elaborated in non-specialist accounts of the experiment and identifies original, key-explanatory elements which characterize them. In so doing, the paper presents a reflection on the processes of transformation and adaptation implied by the circulation of knowledge - which features as a productive process in its own right - and shows which further insights a focus on explanation can offer to the current historical researches on science communication.

History of public communication of science; Popularization of science and technology; Public understanding of science and technology

Explanation in Science traditionally belongs to the research interests of several scholarly disciplines; as attested by the book Explanations: Styles of Explanation in Science, published in 2004 and edited by John Cornwell, the topic can be taken as pivot around which contributions from philosophy, mathematics, physics and linguistic can be articulated. According to the authors of the book, the exploration of the 'mechanisms, validity, connections and disparities between various forms and style of explanation employed in science' can provide a significant contribution to the public understanding of science [Cornwell, 2004, p. xviii]. Moreover, explanations in science provided fruitful material to researches focused on the rhetoric of popular science, its role and place within the scientific discourse [Fahnestock, 1986; Myers, 2003; Schickore, 2001; Charney, 2003; Paul, 2004] and to the (still on-going) debate concerning the relation between explanation and understanding from a philosophical perspective [de Regt, 2013].

However, if explanation is considered as a mean for making science contents accessible to a wide audience a still provoking insight into the matter is provided by Albert Einstein in the forward to Lincon Barnett's popular book on relativity:

\footnotetext{
"Anyone who has ever tried to present a rather abstract scientific subject in a
} popular manner knows the great difficulties of such an attempt. Either he 
succeeds in being intelligible by concealing the core of the problem [...] or else he gives an expert account of the problem, but in such a fashion that the untrained reader is unable to follow the exposition and becomes discouraged from reading any further" [Einstein, 1948].

And yet, over the time, several attempts have been made in order to go through the Scylla and Charybdis abyss of deceit and discouragement depicted by Einstein, who himself put significant efforts in this respect. This paper investigates such attempts in the case of a specific episode of $20^{\text {th }}$ Century physics - the crucial experiment for the discovery of parity violation in 1956. In particular, four accounts of the experiment taken from different editorial categories -a peer-review journal, a popular science book, an encyclopedia and a newspaper articles - are analyzed and compared: if the transmission of knowledge implies transformation, translation and adaptation, the aim is to identify the processes according to which these processes happen and to understand if, why and how the circulation of knowledge can be considered a productive process in its own right [Secord, 2004]. In this respect, the study here developed identifies original, key-explanatory elements which emerge and stabilize when the results starts to circulate outside the inner circle of the peers; in so doing, the study shows that, for the understanding of the circulation of knowledge, issues of transformations instead of degradation and of interchanges instead of question of origins become more prominent and significant [Hilgartner, 1990; Beer, 1990].

The specific episode chosen as case study is the experiment performed by the team led by Chien-Shiung $\mathrm{Wu}$ - known also as Madame $\mathrm{Wu}$ or the first lady of physics — in the late 1956 and early 1957 at the US National Bureau of Standards on "parity violation". This experiment made to collapse the universal assumption of physics that the symmetry of parity was respected by all the known interactions between particles (electromagnetic, strong and weak) by showing that, at least for the weak, the assumption fails.

Section 2 is devoted to the introduction of the historical episode and its main characters. In section 3 I will develop the analysis by considering how the experiment and its results have been presented in the original paper, in newspapers, pedagogical-oriented accounts, popular science books and literary fiction. In section 4 I will speculate on the meaning of tracing an history of explanation in science communication and how the study of the explaining strategies opens up to new possibilities for historical researches.

On the discovery of parity violation and its explanations
Symmetry (or asymmetry) properties of natural objects and species have long been classificatory criteria used by science in the study of the Nature; at the same, human artefacts such as mosaics are meaningful exhibit of the fruitful partnership established between mathematical symmetry transformations and aesthetic criteria of beauty.

However, a third, specific meaning of symmetry is particularly interesting for the present paper: the one according to which, symmetry relates to indistinguishability. ${ }^{1}$ An early example of this use goes back to Galileo Galilei and

\footnotetext{
${ }^{1}$ For a wide discussion of the different uses of symmetry in physics beyond the one here considered, see Brading and Castellani [2003].
} 
the thought experiment reported his Dialogue Concerning the Two Chief World Systems of 1632: in it, the scientist tries to persuade the reader that, if she/he were in the hull of a windowless ship looking at the flight of a fly, at the spilling of the water from a recipient, walking, jumping, throwing an object to a companion... she/he wouldn't be able to distinguish whether the vessel is at rest on the dock or whether is moving at a constant speed. In fact, all the effects are going to be the same in both the two situations which turn to be indistinguishable. The invariance for relative motion transformations or "Galileo transformation" - a symmetry property of the systems moving at constant speed - is today so embedded in the common perception to make sometimes hard to believe that there has been a time in which the performance of such a test - even mental as it was had been necessary. ${ }^{2}$

Analogously, the symmetry for parity transformations possessed the same persuasive character of plausibility and self-evidence which made the search for a proof almost unessential: however, in this case, suggestions for a test quickly turned into a series of "crucial experiments" which made to collapse an assumption which had been so easy to accept. ${ }^{3}$

Which are, in this case, the two situations to compare and/or the test experiment(s) which are to be performed?

The accounts discussed in the present paper make a point in answering these questions, digging out and conveying that flavor of immediateness and plausibility which makes the assumption on parity conservation sounding acceptable.

Nevertheless, the question is here momentarily postponed in favor of a recollection of the events which revolved around the "downfall of parity".

That the stakes was high for the scientific community is witnessed by a phrase of the famous physicist Wolfgang Pauli who linked the meaning of this symmetry no less than to the handedness of god:

"I do not believe that the Lord is weak left-hander, and I am ready to bet a high sum that the experiments will give a symmetrical distribution of electron". ${ }^{4}$

In a time of not instantaneous global communications, Pauli wrote these words in Zurich on 17 January 1957 without being aware that, during a press conference held at the Columbia University two days earlier, a group of researchers presented the results of a series of experiments which showed that, following the theological metaphor, god is really 'left-handed', at least in one of its four fundamental manifestations, the so called weak interactions.

The first experiment has been performed by a team led by the Sino-American physicist Chien-Shiung Wu in the last months of 1956 at the National Bureau of

\footnotetext{
${ }^{2}$ The thought experiment is also referred to as "Galileian invariance", "Galileian relativity principle" or even "Galileo's ship" [Galilei, 1632/2001, day four]. Wigner argues that Galileo still makes an implicit use of symmetry principles while the first one who explicitly assumed them in the derivation of a theory has been Albert Einstein in the theory of special relativity [Wigner, 1967].

${ }^{3}$ The parity operator was introduced in 1927 by the theoretical physicist, engineer and mathematician Eugene Paul Wigner [Wigner, 1927]. Differently from the Galileo transformations, which involve space and time coordinates, the parity transformations involve only space coordinates.

${ }^{4}$ Letter from Pauli to Weisskopf. The episode is reported in Tung-Mow Yan [1992].
} 
Standards in the United States: ${ }^{5}$ working on an hypothesis advanced by the theoretical physicists Lee and Yang in '56 [Lee and Yang, 1957], Wu and collaborators caught the effect by analyzing the beta decay of polarized Cobalt nuclei. Accordingly to the fame of $\mathrm{Wu}$ as extremely fine experimentalist, accustomed at providing data to a high degree of reliability, she did not give any formal scientific communication before the 15th of January 1957. On that day, the editor of the prestigious journal Physical Review received the letter Experimental Test of Parity Conservation in Beta Decay where the results were announced [Wu et al., 1957]. During the Christmas holidays of '56, a second group of researchers of the same University of $\mathrm{Wu}$ (the Columbia University) - Richard Garwin, Leon Lederman, Marcel Weinrich - heard rumors about the ongoing experiment and went immediately into action: in the timeframe of a single feverish weekend they succeeded in achieving the same result by performing a different type of experiment. On January 1957 the 15th, the editor of the Physical Review received a second letter - Observations of the Failure of Conservation of Parity and Charge Conjugation in Meson Decays: the Magnetic Moment of the Free Muon — which attested that the same effect had been detected by analyzing the decay of free muons. At the beginning of this second letter the authors remark that confirmation of Lee and Yang's proposal "in the form of preliminary results on beta decay of oriented nuclei by Wu et al. reached us before this experiment was begun" [Garwin, Lederman and Weinrich, 1957]. The two letters have been published, one after the other, in the 105 Volume of Physical Review, 15 February issue (Letters to the Editor). In the afternoon of the 15th January, the two groups gave the public announcement in the mentioned press conference and the day after the New York Times carried a front-page headline, "Basic Concept in Physics Reported Upset in Tests". ${ }^{6}$ Two days after, on the $17^{\text {th }}$ January, a third letters to the Physical Review Nuclear Emulsion Evidence for Parity Nonconservation in the Decay Chain $\pi^{+-} \mu^{+-} \mathrm{e}^{+-}$announced that a third group formed by Jerome Friedman and Valentine Telegdi from the University of Chicago, detected the effect by using a third experimental technique, the nuclear emulsions [Friedman and Telegdi, 1957a]. ${ }^{7}$ Before the summer, both the first and third research group sent further confirmation of their tests [Ambler et al., 1957; Friedman and Telegdi, 1957b] and, in October, a further test on parity violation was successfully performed with the new emerging detector for particle physics: the bubble chamber [Eisler et al., 1957]. With a highly unusual speed - also a sign of the strong impression aroused by the whole episode in the scientific community — those Lee and Yang who dared to formulate the hypothesis and launching the experimental challenge were awarded with the Nobel Prize on $11^{\text {th }}$ December of the same year 1957.

From the time when the researchers announced their results to date, explanatory accounts on parity and its violation quickly flourished outside from the peer-review journals into encyclopedias, popular science books, magazines, periodicals of scientific institution, web-portal for scientific curiosity: in 1993, one of the members of the second group, the Nobel Prize Leon Lederman, in his popular science book The God Particle: If the Universe Is the Answer, What Is the Question?, co-authored by the science writer Dick Teresi [Lederman and Teresi, 1993], told his

\footnotetext{
${ }^{5}$ As suggested by her nickname, $\mathrm{Wu}$ has been an exceptional character of female scientist about which not much literature is available. Probably the most comprehensive account is Chiang [1996].

${ }^{6}$ The day after, on $17^{\text {th }}$ January, an editorial "Appearance and Reality" was published too.

${ }^{7}$ Initially request for further specification, this last letter was still published on the 105 Volume of the journal, but on the 1 March Issue.
} 
own side of the story occurred in the first days of ' 57 and, by doing so, he provided one of the most extensive account of what the symmetry of parity is, what its violation mean and how these concepts can be explained to a wide audience.

Because of the historical role played by the authors and of the difference in genre, Wu's original account and Lederman's account of Wu and his experiment are the two special terms of reference for the analysis carried out in the next section. However, the study will be widened by considering also pedagogically oriented accounts of the experiments, newspaper articles.

Tracing a history of parity violation through its explanations

\subsection{The first communication(s): Wu's original paper and New York Times article}

Coherently with its character of "letter to the editor", Wu's account of the experiment is extremely synthetic: after a reference to the hypothesis advanced by Lee and Yang, $\mathrm{Wu}$ goes straight to the phenomena under investigation and to the results of the experiment:

\begin{abstract}
"In beta decay, one could measure the angular distribution of the electrons coming from beta decays of polarized nuclei. If an asymmetry in the distribution between $\theta$ and $180^{\circ}-\theta$ (where $\theta$ is the angle between the orientation of the parent nuclei and the momentum of the electrons) is observed, it provides unequivocal proof that parity is not conserved in beta decay. This asymmetry effect has been observed in the case of oriented Co60." [Wu et al., 1957, p. 1413].
\end{abstract}

Addressed to a specialist audience, the argument takes for granted an amount of technical knowledge concerning the angular distribution, the parity operator, beta decay of the 60-Cobalt nuclei, the polarization: the rest of the paper focuses on a detailed analysis of the experimental setting which was the very challenging aspect for experimental physicists of the time. Then, the numerical values and their possible interpretation as a confirmation of the effect is reported in a graph.

For the purposes of the present paper, it is meaningful to consider the way how this sophisticated result has been communicated to the public just the day after: in fact, the pages of the New York Times not only report, as expected, about the upsetting character of the discovery which made collapse an "assumed belief", about extremely young scientists involved but also about the parity principle itself and its detected violation:

\begin{abstract}
"Assume that one motion picture camera is photographing a given set of actions and that another camera simultaneously is photographing the same set of actions as reflected in a mirror. If the two films are later screened, a viewer would have no way, according to the principle of parity, of telling which of the two was the mirror image. The recently completed experiments indicate that there is a way of determining which of the two images is the mirror image".
\end{abstract}

The mental experiment - which can be found essentially unaltered in many contemporary accounts - catches the essence of the concept by indicating that parity is violated whenever there is a way of distinguishing between reality and its 
mirror image. If the observer has no way to get to this awareness, parity is conserved.

The comparison of the two articles reveals more than a smart strategy: in the compact form of the technical language used by Wu the violation of parity stays in strict relation to the specific experiment - parity violation is an "asymmetry in the distribution of the electrons emitted in a beta-decay of oriented Cobalt nuclei" while in the openness of the plain language used in the newspaper the concept is elaborated to the more general, but still meaningful level, and includes a variety of situations which go beyond the experiment and particle physics itself. More than this, it bridges the principle of parity to a so self-evident situation, to make it hard for everyone to believe that such a principle shouldn't hold in all possible, imaginable situations.

Which part does the experiment play in the possibility of distinguishing between reality and its mirror image? How the "new knowledge" produced by the experiment itself can be expressed?

Rather than a "degradation in the quality of the message", in the newspaper article there is a plausible loss of the amount of the initial information encrypted in the technical language: filling this "gap" implies to develop wider account aimed at building strategies and developing literacy around the experiment itself and its place within science. In other words, it means to go through the tricky abyss depicted by Einstein, as it will be for the writings discussed shown in the next sections.

\subsubsection{Semi-technical level: pedagogical-oriented accounts}

The use of a mirror as an explanatory tool marks the "exit" from the fully-specialist language into a semi-technical level represented by pedagogically oriented accounts of various types which can be textbooks, entries of scientific encyclopedia and articles in science focused magazines.

An example of this use is provided by the 'Stanford Encyclopedia of Philosophy' which frames the discovery of parity violation within the relationship between crucial experiments and competing theories [Franklin and Perovic, 2015]. The experiment is quoted for its role in solving the " $\tau-\theta$ " puzzle: according to a set of criteria (mass and lifetime) the two elementary particles (tau and theta) appeared to be the same, whereas on another set of criteria (spin and intrinsic parity) they appeared to be different. Lee and Yang [1957] realized that the problem would be solved, and that the two particles would be different decay modes of the same particle, if the symmetry parity - at the time an "assumed belief" - would have been violated in particle decays. Moreover, they remarked that no evidences supported the conservation and proposed a series of experiment to test it. $\mathrm{Wu}$ 's experiment was one of those.

The explanation of Wu experiment on the Encyclopedia does not replicate the one in the original paper, neither the one in the newspaper: it builds around a new visual element reported in Figure 1. The figure contains a "representation" of the phenomenon studied by $\mathrm{Wu}$ - the beta-decay of polarized Cobalt nuclei observed in a mirror. It is not Wu's experimental setting, but an extrapolation of it. 


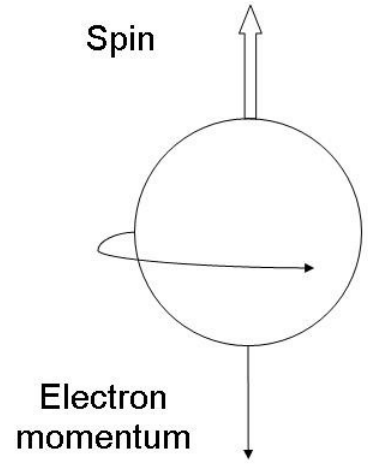

REAL SPACE

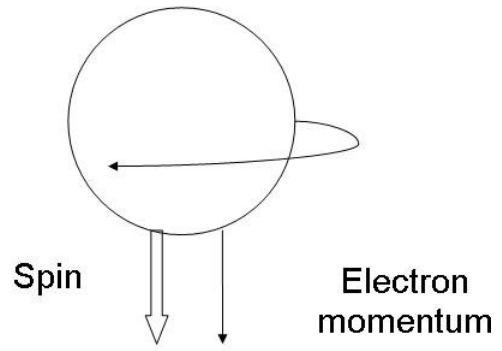

MIRROR SPACE

Figure 1. Nuclear spin and momentum of the decay electron in decay in both real space and in mirror space.

A nucleus spinning from left to right is represented with a sphere and vector pointing up-ward (spin). While it spins, it emits electrons in one direction only: down-ward. If the same scene is observed through the mirror, the rotation is left to right and, accordingly, the spin inverts its direction. However, the direction of the emitted electrons is still downward. According to this construction, parity is violated because the process in the real space is different from its mirror image. If the emitted electrons would have been observed in both directions the two situations would be identical and parity conserved. The technical notion of "distribution" is absorbed within the momentum vector which indicates the "preferential direction" of the emitted electrons.

The use of the mirror is also the reason for the coming into being of the traditional use of the naming the symmetry of parity as the "left-right" symmetry which turns to be not particularly fortunate: one could conclude that the process violates parity if "the left is not equal to right" or if, when observed through the mirror, the left part does not coincide with the right part instead of realizing that the difference at issue concerns the distinction between reality and its mirror-image.

\subsubsection{Parity violation in popular science writing: Lederman and Teresi book The God Particle}

Within this long-breath narrative aimed at showing how the inner sense of the whole XXth century physics culminates into the search for the 'God Particle', the authors dedicates an Interlude of almost twenty pages to the discovery of parity violation. ${ }^{8}$

The Interlude is a complex piece of writing where the overall narration is displayed, at least, along three distinct levels, that are: - the personal story of one of the authors who was directly involved in the events of '57; - the global reflection on the nature of scientific inquiry; - the conceptual meaning of parity and its violation and the experimental proofs.

\footnotetext{
${ }^{8}$ Interlude $C$ between chapter 6 and 7 out of 9 .
} 
Along this three level the authors provide an orchestration of all the elements currently used in the explanation (the mirror, the spinning objects, the theological metaphor on the handedness of God, ...) with a number of new ones concerning the analogy between micro-world and macro-world, the illustration of sociological aspects of the discovery and the enrichment of the vocabulary.

In so-doing they come to the more general elaboration of the concept of parity violation mechanism which can be equally used for both of the experiments.

Addressing common sense and Nature of Science. From the very first lines of the Interlude, the authors address reader's common sense by pointing out that none of us, by raising her/his right-hand in front of a mirror, would expect her/his mirror image to raise the right hand too; nevertheless, 'an equivalent act did occur with a fundamental particle called muon'. Besides strengthening the sense of curiosity in the reader the example conveys a non-mathematical sense of parity violation in terms of macroscopic objects which are the mirror and the person: it is this "rough explanation" which will be the subject of a knowledge transformation developed along the whole chapter.

However, before pointing straight to this goal, the authors take the opportunity of expanding the new vocabulary and, by doing so, propose to the reader general considerations concerning the nature of scientific inquiry. They say that parity is a symmetry and that symmetry is a very central concept in physics: physicists love symmetry because it has a mathematical and intuitive beauty (Taj Mahal or Greek Temples are examples of symmetry in art); consequently, by virtue of this belief, the laws of nature have been crafted in order to incorporate a rich set of symmetries that were supposed to be absolute and perfect ... at least before January 1957.

Formalizing knowledge, setting the question and creating expectation. It is in the section "Experiment in the mirror" that the authors start to refine the notion of parity violation: they give, for the sake of the record, the appropriate mathematical statement and suddenly pose the central question for understanding parity, that is: if one wall of the lab is a mirror, and scientists in the lab are carrying out experiment "Is there is any way of deciding which is the true lab and which is the mirror lab?". The central question remains unanswered for some time because another kind of reflections, of historical nature, are developed: since the "downfall of parity" is the quintessential example of how physicists think, proceed and adapt to shocks, then it is worth narrating the events occurred around January 1957. So, in the section "The Shangai Cafe", we enter a story which draws upon a specific research context (the one of the Columbia University) where Lederman has been one of the principal actors. The Friday lunch at the Shanghai Cafe was a 'must' for the members of the Columbia University which gathered outside the office of Professor Tsung Dao Lee. But that Friday, 4 January 1957, has been special since, after composing the lunch menu in his peculiar manner, Lee plunges into the general conversation by saying excitedly: "Wu telephoned and said her preliminary data indicated a huge effect!". Madame Wu is introduced here as an extremely fine experimentalist who, accustomed at providing data to an high degree of reliability, was postponing the publication of her results pursued in the previous weeks at the at the National Bureau of Standards in the United States. The announcement caused a sensation among the listeners and Lederman, as he 
himself reports, was among these. The personal story brings the overall narration to the point that a careful explanation of parity in the context of particle physics can no longer be postponed: so, the scenario quickly turns back into the laboratory with a mirror-wall and the explanation of the experiment that $\mathrm{Wu}$ was performing is presented.

The first experiment: fixing the point, the story from outside. The object of study was the Cobalt-60 nuclei cooled at extremely low temperatures in an external magnetic field: in these conditions all they can do is spinning and, by virtue of the weak interaction to which they are subjected, changing into a nucleus of nickel, emitting a neutrino and a positive electron (a positron). "What one 'sees' is that is that the cobalt nucleus suddenly shoots off a positive electron" (the so called, beta-decay). To combine observations in the directions of the spinning emitting nucleus and of the propagating emitted electrons is, as the authors remark, the real ingenious proposal of Lee and Yang and the key-element for understanding. In fact, when the phenomenon is observed through the mirror an essential aspect comes out: if a spinning nucleus would emit an equal numbers of positrons in the up and down directions, the mirror image of the process would be indistinguishable from the real process (occurring in the lab). What Wu discovered (the "huge effect"), is that there is a strong preference in the directions of the emitted positrons such that "one could tell which was the real experiment, which was the mirror image". The notion of parity violation as emerges from Lederman's description of $\mathrm{Wu}$ 's experiment is transformed from the one given at the beginning of the chapter: there, parity violation in the macro-world meant, eventually, that a person raising an hand in front of the mirror sees the mirror image raising the same hand. In the new form, instead, parity violation (in micro and macro-worlds) means that there is a way of being aware whether what is observed is reality or its mirror image, i.e. if there is an element of knowledge allowing the experimenter to distinguish between the two situations ("which was the real experiment, which was the mirror image"). On the contrary, when she/he has no way of making such a distinction, it is said that parity (or symmetry $\mathrm{P}$ ) is conserved. In Wu's experiment this element is the preferential direction of the emitted positrons.

Ten pages after the beginning of the Interlude the refinement of the 'rough explanation' of parity violation into a refined concept has been carried out. In the last section of the book, a schema will put this knowledge in full swing.

The second experiment: the story from inside. Section "The experiment", in fact, is a detailed account of the second type of experiment performed by Lederman, Garwin and Weinrich in the week-end immediately after the Friday's lunch of 4 January 1957. The idea of the experiment was to select traveling muons with a known direction of the spin and observe them decaying into an electron and some neutrinos: the situation is the same that $\mathrm{Wu}$ organized by cooling cobalt- 60 nuclei to extremely low temperatures in a magnetic field, with the difference that the expected asymmetry was in the direction of the emitted electrons (instead of positrons). The muons themselves were the product of a decay of pions escaped by a target bombarded with high energy protons at the Columbia accelerator (Nevis Lab in Irvington-on-Hudson). 
The following figure (Figure 2), reported at page 262, provides a schema for both the experiments:
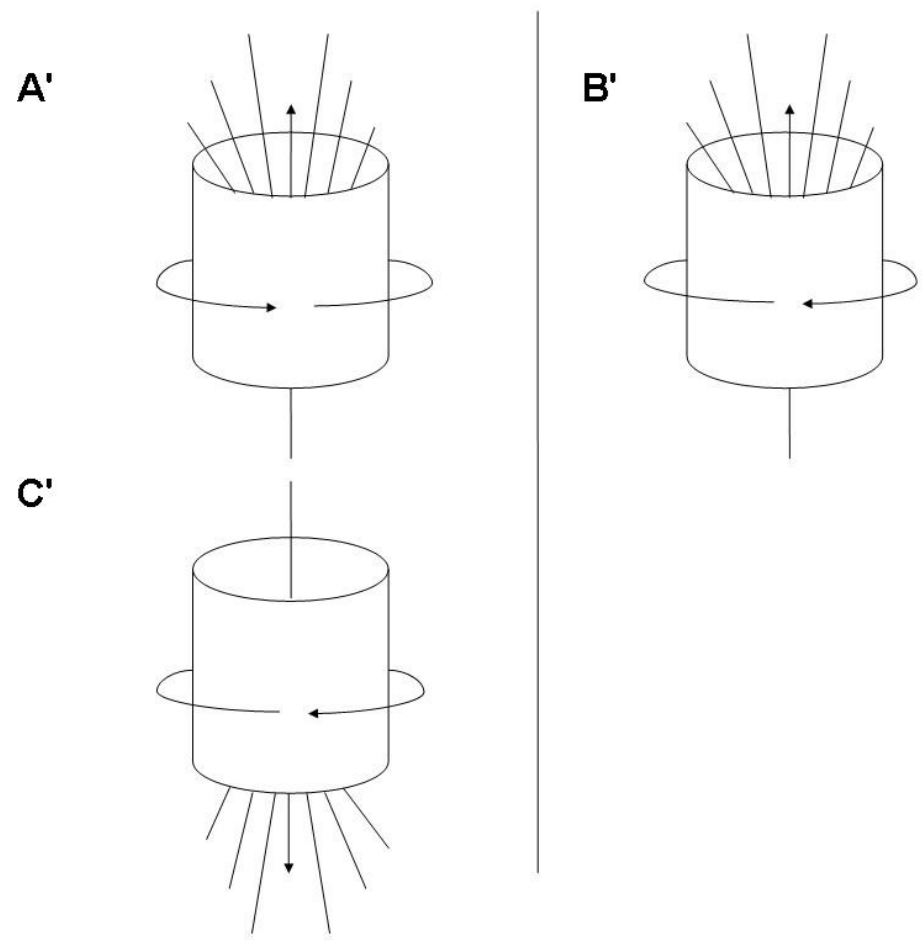

Figure 2. Object $\mathrm{A}^{\prime}$ portrays a decaying muon. The spin axis now has an arrow indicating the direction of emission of electrons. The rays signify that the electrons strongly prefer "righthandedness". Object B', the mirror image, is a left-handed decaying muon. If experiment shows that all muon decays are right- handed, then $B^{\prime}$ does not exist in nature. For example, turning $\mathrm{A}^{\prime}$ upsidedown $\left(\mathrm{C}^{\prime}\right)$ does not replicate $\mathrm{B}^{\prime}$. Mirror symmetry is violated'.

However, this section do not tell us only about physical/technical contents or details: since the episode is considered by the authors as a paradigmatic case of how physicists proceed in their research, the level of the personal story of the researchers is regained and told in detail. Indeed, the majority of the section focuses on recounting how the news of the "big effect" detected by Wu gave Lederman the strength of regaining their old idea; how this idea was refined in the mind of the researches while he was driving on the heavy traffic of the Saw Mill River Parkway; how Lederman shared the new idea with a colleague, Richard Garwin, to get help from him; how the two rushed in the Lab where a PhD student, Marcel Weinrich, was working at the dissertation, disassembled his apparatus and dragged him into one of the most exciting discovery of the century. The Interlude ends with a detailed description of how, hour after hour, starting on Friday 8 pm to the early Sunday morning, the three researches set up the apparatus, took and analyzed data, struggled with the tension to rush and the need to be careful, and organized themselves for some hours of sleep. By developing a the explanation around the central question "Is there is any way of deciding which is the true lab and which is the mirror lab?", Lederman and Teresi go back to the mental experiment proposed in the New York Times and provide an extensive answer based on the experiment. 
The present study provided an attempt to delineate a history of explanation in science communication by following and analyzing the different forms of explanation on a famous episode in the history of 20th Century physics - the crucial experiment for the discovery of the parity violation in 1956.

The aim brought to investigate the "expository continuum" [Hilgartner, 1990] by reading across usually distinct categories as research, educational and popular accounts [Staley, 2013] and to identify stable and variable elements within the explanatory process.

In particular, as soon as the accounts escape the inner circle of the specialists, a specific element - the mirror - is regularly used as a key-element for the explanation of the concept. At the same time, coherently with their different scopes, the accounts here considered build differently upon the same object: the mirror is a mean to provide an insightful grasp to the concept in the case of the New York Times article, to introduce elements of formalized knowledge and modelling (spinning-objects) in the case of the Encyclopedia, to widen the explanatory schema to a large extent, shifting from the macro to the micro-world phenomena, introducing a new vocabulary, intertwining historical passages and sociological insights in the case of Lederman's account. The thickening of the explanation develops through diverse genres of texts: a newspaper article, an entry in a traditional-style Encyclopedia where entries are written and maintained by experts in the field and a representative example of popular science books of the late $20^{\text {th }}$ Century.

On a more general level, the focus on explanations allowed to approach a well-known, milestone episode in the history of $20^{\text {th }}$ Century physics, studied even in the details of its historical anticipations, ${ }^{9}$ by identifying "new" agents and contingencies - the press conference of the 15th afternoon and the contents of the article, historical actors such as Lederman observed under the new perspective of participant and narrator of his and Wu's experiment - and making them to become an integral part of the process under investigation. Last insight offered by the paper is a reflection how much the understanding of some factors - such as the feeling of "self-evidence" and "down-fall" of an assumption or the revolutionary nature of a suggestion - might be regained by the looking through the folds of different types of explanation. 'Further Experiments on $\beta$ Decay of Polarized Nuclei'. Physical Review 106 (6), pp. 1361-1363. DOI: 10.1103/physrev.106.1361.

Beer, G. (1990). 'Translation or Transformation? The Relations of Literature and Science'. Notes and Records of the Royal Society 44 (1), pp. 81-99. DOI: 10.1098/rsnr.1990.0006.

Brading, K. and Castellani, E. (2003). Symmetries in Physics, Philosophical Reflections. Cambridge University Press.

Charney, D. (2003). 'Lone Geniuses in Popular Science: The Devaluation of Scientific Consensus'. Written Communication 20 (3), pp. 215-241. DOI: $10.1177 / 0741088303257505$.

\footnotetext{
${ }^{9}$ A detailed historical account of the parity violation experiments can be found in A. Franklin [1986] and Franklin [1990].
} 
Chiang, T. C. (1996). Madame Wu Chien-Shiung: The First Lady of Physics Research. World Scientific.

Cornwell, J. (2004). Explanations: Styles of Explanation in Science. Oxford University Press.

de Regt, H. W. (2013). 'Understanding without Explanation'. Special section Studies in History and Philosophy of Science Part A 44 (3), pp. 505 -538.

Einstein, A. (1948). Foreword to The Universe and Doctor Einstein by Lincoln Barnett. Dover.

Eisler, F., Plano, R., Prodell, A., Samios, N., Schwartz, M., Steinberger, J., Bassi, P., Borelli, V., Puppi, G., Tanaka, G., Woloschek, P., Zoboli, V., Conversi, M., Franzini, P., Mannelli, I., Santangelo, R., Silvestrini, V., Glaser, D. A., Graves, C. and Perl, M. L. (1957). 'Demonstration of Parity Nonconservation in Hyperon Decay'. Physical Review 108 (5), pp. 1353-1355. DOI: 10.1103/physrev.108.1353.

Fahnestock, J. (1986). 'Accommodating Science: The Rhetorical Life of Scientific Facts'. Written Communication 3 (3), pp. 275-296. DOI: $10.1177 / 0741088386003003001$.

Franklin, A. (1986). The Neglect of Experiment. Cambridge, U.K.: Cambridge University Press.

- (1990). Experiment, right or wrong. Cambridge, U.K.: Cambridge University Press.

Franklin, A. and Perovic, S. (2015). Experiment in Physics. Appendix 1: The Discovery of Parity Nonconservation. Spring 2015 Edition. Ed. by E. N. Zalta. The Stanford Encyclopedia of Philosophy. URL: http://plato.stanford.edu/entries/physics-experiment/app1.html.

Friedman, J. I. and Telegdi, V. L. (1957a). 'Nuclear Emulsion Evidence for Parity Nonconservation in the Decay Chain $\pi^{+-} \mu^{+-} \mathrm{e}^{+-}$. Physical Review 105 (5), pp. 1681-1682. DOI: 10.1103/physrev.105.1681.2.

- (1957b). 'Nuclear Emulsion Evidence for Parity Nonconservation in the Decay Chain $\pi^{+} \rightarrow \mu^{+} \rightarrow \mathrm{e}^{+\prime}$. Physical Review 106 (6), pp. 1290-1293. DOI: $10.1103 /$ physrev.106.1290.

Galilei, G. (1632/2001). Dialogue Concerning the Two Chief World Systems: Ptolemaic and Copernican. Ed. by S. J. Gould. Modern Library.

Garwin, R. L., Lederman, L. M. and Weinrich, M. (1957). 'Observations of the Failure of Conservation of Parity and Charge Conjugation in Meson Decays: the Magnetic Moment of the Free Muon'. Physical Review 105 (4), pp. 1415-1417. DOI: $10.1103 /$ physrev. 105.1415.

Hilgartner, S. (1990). 'The Dominant View of Popularization: Conceptual Problems, Political Uses'. Social Studies of Science 20 (3), pp. 519-539. DOI: $10.1177 / 030631290020003006$.

Lederman, L. M. and Teresi, D. (1993). The God Particle: If the Universe Is the Answer, What Is the Question? New York, U.S.A.: Dell Publishing.

Lee, T. D. and Yang, C. N. (1957). 'Question of Parity Conservation in Weak Interactions'. Physical Review 106 (6), pp. 1371-1371. DOI: $10.1103 /$ physrev.106.1371.

Myers, G. A. (2003). 'Discourse studies of scientific popularization: questioning the boundaries'. Discourse Studies 5 (2), pp. 265-279. DOI: $10.1177 / 1461445603005002313$.

Paul, D. (2004). 'Spreading Chaos: The Role of Popularizations in the Diffusion of Scientific Ideas'. Written Communication 21 (1), pp. 32-68. DOI: $10.1177 / 0741088303261035$. 
Schickore, J. (2001). 'The Task of Explaining Sight - Helmholtz's Writings on Vision as a Test Case for Models of Science Popularization'. Science in Context 14 (03), pp. 397-417. DOI: 10.1017/s026988970100014x.

Secord, J. A. (2004). 'Knowledge in transit'. ISIS 95 (4), pp. 654-672. DOI: $10.1086 / 430657$. PMID: 16011300.

Staley, R. (2013). 'Trajectories in the History and Historiography of Physics in the Twentieth Century'. History of Science 51 (2), pp. 151-177. DOI: $10.1177 / 007327531305100202$.

Wigner, E. (1927). 'Einige Folgerungen aus der Schrödingerschen Theorie für die Termstrukturen'. Zeitschrift für Physik 43 (9-10), pp. 624-652. DOI: $10.1007 / \mathrm{bf} 01397327$.

- (1967). Symmetries and Reflections. U.S.A.: Indiana University Press.

Wu, C. S., Ambler, E., Hayward, R. W., Hoppes, D. D. and Hudson, R. P. (1957). 'Experimental Test of Parity Conservation in Beta Decay'. Physical Review 105 (4), pp. 1413-1415. DOI: 10.1103/physrev.105.1413.

Yan, T. (1992). 'Professor C.N.Yang Impact on Physics'. Chinese Journal of Physics 30 (7), pp. 1025-1030.

Author

Eugenio Bertozzi is currently a scholar in residence at the Research Institute for the History of Science and Technology of the Deutsches Museum in Munich and adjunct professor at the University of Bologna. He has been Research Fellow of the Humboldt Foundation at the Europa-Universität in Flensburg and scientific director of the Italian science centre 'Museo dela Bali'. He received his Ph.D. in 2010 from the University of Bologna. E-mail: eugenio.bertozzi2@unibo.it.

\section{How to cite}

Bertozzi, E. (2017). 'Toward a history of explanation in science communication: the case of Madame Wu experiment on parity violation'. JCOM 16 (03), A10. 